$\Rightarrow$ LEARNING AND MEMORY

\title{
A subcortical reorganizer
}

Some principal neurons in the hippocampus termed place cells preferentially fire when an animal is in a specific location within an environment. Under certain conditions, place cell responses can be reorganized, such that they preferentially respond to different locations. Indeed, with spatial reward learning, place cell responses at a population level may change to overrepresent locations near to a new reward. The mechanisms that underpin this plasticity remain unclear, but a new study shows that inputs from the locus coeruleus (LC) to the hippocampal CA1 region have an important role in reorganizing place cell responses in mice undergoing such learning.

To assess changes in place cell responses, the authors measured calcium signals in the dorsal CA1 of head-fixed mice by two-photon microscopy while the animals performed a spatial reward learning task on a 2-metre treadmill; the treadmill included various tactile cues to allow the animal to determine its spatial location. In this task, the mice were presented with a water reward in one area (reward zone 1) for 3 days; the location of the reward was then changed so that it occurred

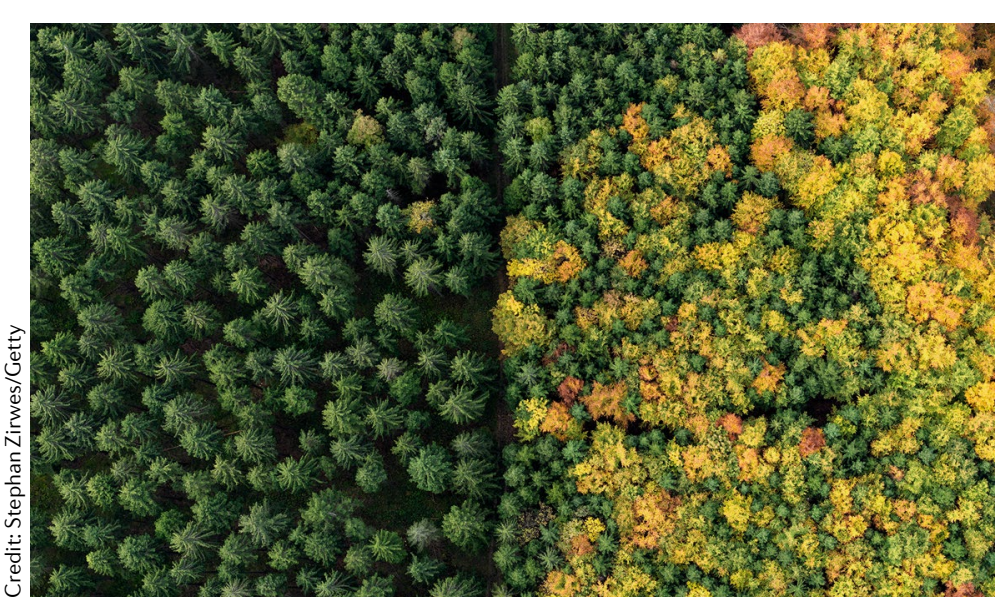

the reward was encountered. In relation to behaviour, the authors found that the extent to which the LC-CA1 axon signal and velocity became decoupled was indicative of how well an animal learned the location of the reward in zone 2 . Strikingly, mice that did not learn the new location of the reward continued to exhibit a tight correlation between LC-CA1 axon activity and velocity.

The decoupling of LC-CA1 axon activity and velocity began to manifest itself in the earliest stages of learning the new position of the reward, before reward zone 2 became overrepresented in place cell responses. Thus, the authors next directly tested whether altering activity in LC-CA1 axons could affect place cell reorganization.

They found that optogenetic activation of LC-CA1 axons did not induce acute responses in CA1 place cells. However, when they activated these cells during the early stages of the task when a mouse was in areas spanning reward zone 1 , they could induce an overrepresentation of this reward zone in place cell responses by the last session of that phase of the task. Moreover, when they optogenetically inhibited LC-CA1 axons during learning of the second reward zone, they could decrease the overrepresentation of place cell responses to that reward zone. Interestingly, optogenetic activation of LC-CA1 axons could not affect place cell responses when it was not paired with a reward location or when it was randomly paired with a reward during a non-spatial task (that is, one in which animals could not learn the location of the reward).

Together, these data show that LC-CA1 activity has a key role in reorganizing place cell responses during spatial reward learning.

Darran Yates 